Swarthmore College

Works

$5-14-2011$

\title{
Experimental Characterization of The Weakly Anisotropic CN $X(2) \Sigma(+)+$ Ne Potential From IR-UV Double Resonance Studies Of The CN-Ne Complex
}

J. M. Beames

B. A. O'Donnell

M. Ting

M. I. Lester

Thomas Alex Stephenson

Swarthmore College, tstephe1@swarthmore.edu

Follow this and additional works at: https://works.swarthmore.edu/fac-chemistry

Part of the Physical Chemistry Commons

Let us know how access to these works benefits you

\section{Recommended Citation}

J. M. Beames, B. A. O'Donnell, M. Ting, M. I. Lester, and Thomas Alex Stephenson. (2011). "Experimental Characterization of The Weakly Anisotropic CN X(2) $\Sigma(+)+$ Ne Potential From IR-UV Double Resonance Studies Of The CN-Ne Complex". Journal Of Chemical Physics. Volume 134, Issue 18. DOI: 10.1063/ 1.3586810

https://works.swarthmore.edu/fac-chemistry/11

This work is brought to you for free by Swarthmore College Libraries' Works. It has been accepted for inclusion in Chemistry \& Biochemistry Faculty Works by an authorized administrator of Works. For more information, please contact myworks@swarthmore.edu. 


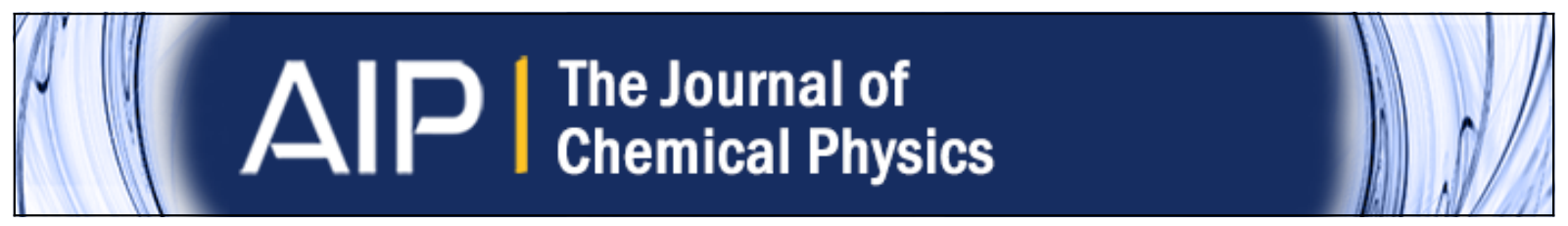

Experimental characterization of the weakly anisotropic CN X 2+ + Ne potential from IRUV double resonance studies of the $\mathrm{CN}-\mathrm{Ne}$ complex

Joseph M. Beames, Bridget A. O'Donnell, Melodie Ting, Marsha I. Lester, and Thomas A. Stephenson

Citation: The Journal of Chemical Physics 134, 184308 (2011); doi: 10.1063/1.3586810

View online: http://dx.doi.org/10.1063/1.3586810

View Table of Contents: http://scitation.aip.org/content/aip/journal/jcp/134/18?ver=pdfcov

Published by the AIP Publishing

\section{AlP Re-register for Table of Content Alerts}

\section{Create a profile. 困 Sign up today!}




\title{
Experimental characterization of the weakly anisotropic $\mathrm{CN} X^{2} \Sigma^{+}+\mathrm{Ne}$ potential from IR-UV double resonance studies of the CN-Ne complex
}

\author{
Joseph M. Beames, ${ }^{1}$ Bridget A. O'Donnell, ${ }^{1}$ Melodie Ting, ${ }^{1}$ \\ Marsha I. Lester, ${ }^{1, a)}$ and Thomas A. Stephenson ${ }^{2}$ \\ ${ }^{1}$ Department of Chemistry, University of Pennsylvania, Philadelphia, Pennsylvania 19104-6323, USA \\ ${ }^{2}$ Department of Chemistry and Biochemistry, Swarthmore College, Swarthmore, Pennsylvania 19081, USA
}

(Received 3 February 2011; accepted 13 April 2011; published online 10 May 2011)

\begin{abstract}
IR-UV double resonance spectroscopy has been used to characterize hindered internal rotor states $\left(n^{K}=0^{0}, 1^{1}\right.$, and $\left.1^{0}\right)$ of the $\mathrm{CN}-\mathrm{Ne}$ complex in its ground electronic state with various degrees of $\mathrm{CN}$ stretch $\left(v_{\mathrm{CN}}\right)$ excitation. Rotationally resolved infrared overtone spectra of the CN-Ne complex exhibit perturbations arising from Coriolis coupling between the closely spaced hindered rotor states $\left(1^{1}\right.$ and $\left.1^{0}\right)$ with two quanta of $\mathrm{CN}$ stretch $\left(v_{\mathrm{CN}}=2\right)$. A deperturbation analysis is used to obtain accurate rotational constants and associated average $\mathrm{CN}$ center-of-mass to Ne separation distances as well as the coupling strength. The energetic ordering and spacings of the hindered internal rotor states provide a direct reflection of the weakly anisotropic intermolecular potential between $\mathrm{CN} X^{2} \Sigma^{+}$and $\mathrm{Ne}$, with only an $8 \mathrm{~cm}^{-1}$ barrier to $\mathrm{CN}$ internal rotation, from which radially averaged anisotropy parameters $\left(V_{10}\right.$ and $\left.V_{20}\right)$ are extracted that are consistent for $v_{\mathrm{CN}}=0-3$. Complementary ab initio calculation of the $\mathrm{CN} X^{2} \Sigma^{+}+$Ne potential using MRCI+Q extrapolated to the complete one-electron basis set limit is compared with the experimentally derived anisotropy by optimizing the radial potential at each angle. Experiment and theory are in excellent accord, both indicating a bent minimum energy configuration and nearly free rotor behavior. Analogous experimental and theoretical studies of the CN-Ne complex upon electronic excitation to the $\mathrm{CN} B^{2} \Sigma^{+}$state indicate a slightly more anisotropic potential with a linear $\mathrm{CN}-\mathrm{Ne}$ minimum energy configuration. The results from these IRUV double resonance studies are compared with prior electronic spectroscopy and theoretical studies of the CN-Ne system. @ 2011 American Institute of Physics. [doi:10.1063/1.3586810]
\end{abstract}

\section{INTRODUCTION}

Many reactions of $\mathrm{CN}$ radicals exhibit unusual kinetic behavior indicative of association of the reactants in a weakly bound radical-molecule complex. This is evident in the reaction kinetics of the $\mathrm{CN}$ radical with hydrocarbons, ${ }^{1-4}$ which have been studied extensively due to the prevalence of cyanogen in the combustion products of fossil fuels and its resulting role in $\mathrm{NO}_{\mathrm{x}}$ chemistry. ${ }^{5}$ In such environments, the $\mathrm{CN}$ radical acts as a pseudo-halogen, reacting rapidly with the gaseous hydrocarbons through addition or hydrogen abstraction. Large rate coefficients in both of these cases suggest that the reactions are barrierless with negative activation energies indicating that pre-reactive complexes are formed, albeit with short lifetimes. ${ }^{2}$ Crossed molecular beam studies of $\mathrm{CN}$ radicals with unsaturated hydrocarbons ${ }^{6}$ have shown that the first step in the reaction pathway is the production of a collision complex, in which the $\mathrm{CN}$ radical adds to the $\pi$ electron density of the hydrocarbon. Although this type of addition mechanism would not be possible with saturated hydrocarbons, theoretical work by Georgievskii and Klippenstein ${ }^{7}$ has predicted a weakly bound van der Waals complex and submerged barrier, separating the pre-reactive complex from product formation, to explain the "strange kinetics" and unusual temperature dependence of the $\mathrm{C}_{2} \mathrm{H}_{6}+\mathrm{CN}$ hydrogen

\footnotetext{
a) Author to whom correspondence should be addressed. Electronic mail: milester@sas.upenn.edu. Tel.: (215) 898-4640. Fax: (215) 573-2112.
}

abstraction reaction. Isolating and studying $\mathrm{CN}$ radical-rare gas (CN-Rg) complexes provides a starting point to study $\mathrm{CN}$ radical-molecule complexes, which have not yet been observed directly.

Thus far, the electronic spectroscopy and excited state predissociation dynamics of $\mathrm{CN}-\mathrm{Rg}$ and $\mathrm{CN}-\mathrm{H}_{2}$ van der Waals complexes have been examined by Heaven and coworkers. ${ }^{8-11} \mathrm{CN}-\mathrm{Ne}, \mathrm{CN}-\mathrm{Ar}$, and $\mathrm{CN}-\mathrm{H}_{2}$ were investigated using laser induced fluorescence (LIF) with initial work on each system focusing on electronic excitation and fluorescence detection in the $\mathrm{CN} B{ }^{2} \Sigma^{+}-X^{2} \Sigma^{+}$region, hereafter referred to as $B-X$ transitions of the complexes. The notation used to denote electronic states of these complexes is not rigorous, but has been adopted in this work and all previous studies to denote states which correlate closely with those of free$\mathrm{CN}$. Rotationally structured CN-Ne $B\left(v_{\mathrm{CN}}=0\right)-X\left(v_{\mathrm{CN}}=0\right)$ excitation spectra were observed by collection of $B$-state fluorescence from the complex itself, with the electronic origin lying close in energy to that of free-CN. This is in stark contrast to the dynamics of $\mathrm{CN}-\mathrm{Ar}$ and $\mathrm{CN}-\mathrm{H}_{2}$ complexes upon $B$ - $X$ excitation: CN-Ar rapidly predissociates into $\mathrm{CN} A^{2} \Pi$ $+\mathrm{Ar}$, such that rovibronic spectra could be observed only by collecting $\mathrm{CN} A-X$ emission. The $\mathrm{CN}-\mathrm{H}_{2} B-X$ excitation spectrum displays no resolvable structure and was attributed to bound-free excitation with subsequent emission from $\mathrm{CN} B$ ${ }^{2} \Sigma^{+}$products. These studies provide a significant amount of information on the excited $B$ (and $A$ ) electronic states of these 
CN complexes; however, little spectroscopic information was obtained on these $\mathrm{CN}$ complexes in their ground electronic state. The present study provides the first infrared spectroscopic study of a $\mathrm{CN}$ radical complex, specifically $\mathrm{CN}-\mathrm{Ne}$, which is utilized to characterize the anisotropy of the $\mathrm{CN} X$ ${ }^{2} \Sigma^{+}+$Ne intermolecular potential. Similar studies of CN-Ar and $\mathrm{CN}-\mathrm{H}_{2}$ will be reported elsewhere.

Alexander and co-workers have generated high level $a b$ initio potential energy surfaces (PES) for $\mathrm{CN}-\mathrm{Ne}, \mathrm{CN}-\mathrm{Ar}$, and $\mathrm{CN}-\mathrm{H}_{2}$, and these were subsequently used in analysis of spectroscopic data, giving insight on the structure and dynamics of the complexes. ${ }^{12-15}$ The $\mathrm{CN} X^{2} \Sigma^{+}+\mathrm{Ne}$ MRCI+Q surface indicates a long-range van der Waals type interaction with $\mathrm{Ne}$ bound to the $\mathrm{CN} X^{2} \Sigma^{+}$radical by just $31.3 \mathrm{~cm}^{-1}\left(D_{\mathrm{e}}\right)$ at an intermolecular separation $\left(R_{\mathrm{e}}\right)$ of $3.75 \AA$. The intermolecular potential exhibits minimal anisotropy, with a barrier to $\mathrm{CN}$ internal rotation of less than $2 \mathrm{~cm}^{-1}$. Another ab initio study of the $\mathrm{CN} X^{2} \Sigma^{+}+$Ne potential by Vrábel et al. yielded a similar angular dependence for the interaction energy using restricted (and unrestricted) MP2 theory, ${ }^{16}$ with $D_{\mathrm{e}}$ $=32.0 \mathrm{~cm}^{-1}, R_{\mathrm{e}}=3.75 \AA$, and a barrier to internal rotation of less than $5 \mathrm{~cm}^{-1}$. The anisotropy of the ground state PES changes for different partners; for example, the $\mathrm{CN} X^{2} \Sigma^{+}$ $+\mathrm{Ne}$ and $\mathrm{H}_{2}$ potentials are relatively flat, ${ }^{14,15}$ with $\mathrm{CN}$ likely to undergo almost free internal rotation, while the $\mathrm{CN} X$ ${ }^{2} \Sigma^{+}+$Ar potential is predicted to be more anisotropic with a bent minimum energy structure. ${ }^{12}$ The minimum energy configuration also changes upon electronic excitation of $\mathrm{CN}$ to the $B^{2} \Sigma^{+}$state, which reverses the direction of the $\mathrm{CN}$ dipole moment, ${ }^{17}$ with $\mathrm{CN}-\mathrm{Ar}$ favoring a linear minimum energy configuration.

Given the shallow nature of the ground and excited state potentials for $\mathrm{CN}-\mathrm{Rg}$ complexes, both infrared and vibronic spectra will likely be dominated by progressions in the low frequency "bending" vibration associated with hindered internal rotor motion. For this reason, this work follows the guide to open shell van der Waals complexes by Dubernet et al. which reduces the effective Hamiltonian for the system to that of the bending (or internal hindered rotor) mode alone. ${ }^{18,19}$ For complexes of $\mathrm{CN}$ in ${ }^{2} \Sigma^{+}$states, which includes its $X^{2} \Sigma^{+}$and $B{ }^{2} \Sigma^{+}$electronic states, this reduced Hamiltonian follows Hund's case (b) approximations and is similar to that of a closed shell system because the unpaired electron spin is only weakly coupled to the nuclear axes. It should be noted that the quantum labels used in such approximations in previous literature are not consistent, and therefore it is useful to define the notation that will be used throughout this work. In such atom-diatom complexes, the diatomic monomer rotational quantum number (denoted as $n$ ) is quantized along the intermolecular axis. ${ }^{18,20}$ This projection quantum number is labeled $K . L$ is the pseudo diatomic quantum number for the end-over-end rotation of the complex, and this can couple with $K$ to form the resultant $N$, which is the total angular momentum (excluding electron spin) and is a good quantum number. Electron spin can only weakly couple with $N$, and therefore is neglected. As a result, $\mathrm{CN}-\mathrm{Ne}$ states will be labeled by their hindered rotor state $\left(n^{K}\right), \mathrm{CN}$ vibrational state $\left(v_{\mathrm{CN}}\right)$, and $\mathrm{CN}$ electronic state $(X$ or $B)$, as needed; the intermolecular stretch $\left(v_{\mathrm{s}}\right)$ is not observed and therefore is generally not included in state labels.

This paper focuses on the $\mathrm{CN}-\mathrm{Ne}$ complex, which has previously been investigated through electronic spectroscopy in the $B$ - $X$ region by Heaven and co-workers. ${ }^{9,11}$ In the present work, an IR-UV double resonance technique is employed to probe the ground electronic state of the $\mathrm{CN}-\mathrm{Ne}$ complex, principally with two quanta of $\mathrm{CN}$ stretch, leading to the observation of several rotationally resolved bands. The rotational structure of these bands and spacings between associated angular states are used to obtain new information on the anisotropy of the $\mathrm{CN} X^{2} \Sigma^{+}+$Ne potential, which is complemented by high level ab initio calculations and compared with previous experimental work on this system.

\section{EXPERIMENTAL AND THEORETICAL METHODS}

An IR-UV double resonance technique is utilized to obtain IR spectra of $\mathrm{CN}-\mathrm{Ne}$ in the $\mathrm{CN}$ stretch overtone region as well as UV spectra in the $\mathrm{CN} B{ }^{2} \Sigma^{+}(\mathrm{v}$ $=0)-X^{2} \Sigma^{+}(\mathrm{v}=2)$ spectral region. As depicted schematically in Fig. 1 the IR laser prepares the $\mathrm{CN}-\mathrm{Ne}$ complexes with two quanta of $\mathrm{CN}$ vibrational excitation. The UV laser then promotes the vibrationally excited $\mathrm{CN}-\mathrm{Ne}$ complexes to the electronically excited $B$ state, from which $B$ - $X$ fluorescence is collected. IR spectra are recorded by fixing the UV laser on a $\mathrm{CN}-\mathrm{Ne}$ electronic transition and scanning the IR laser,

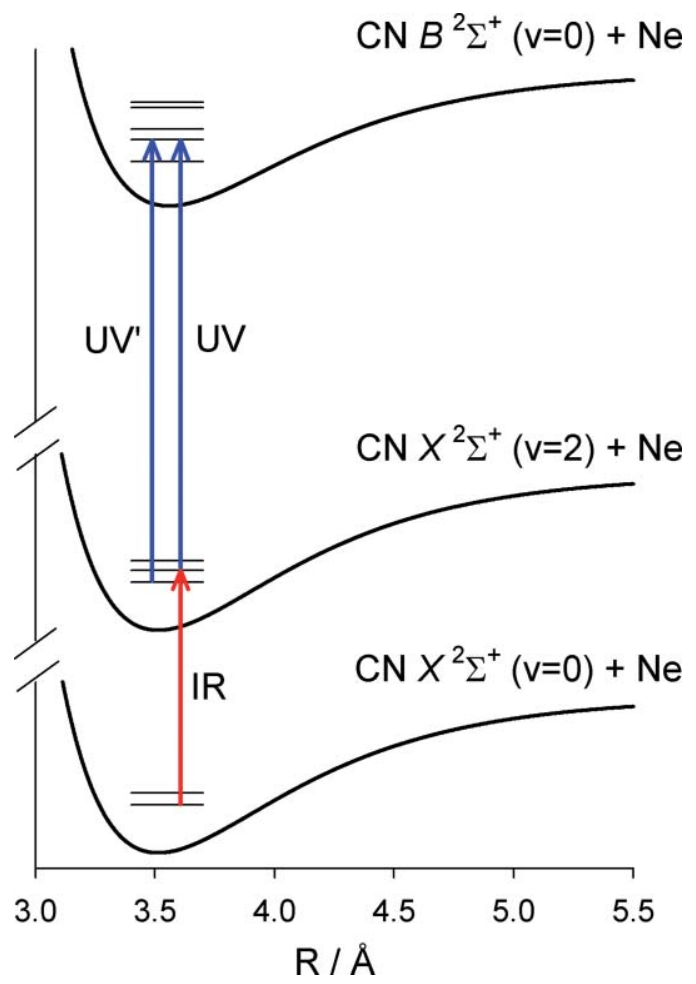

FIG. 1. IR-UV double resonance scheme is shown on schematic radial potentials for $\mathrm{CN}\left(B^{2} \Sigma^{+}, X^{2} \Sigma^{+}\right)+\mathrm{Ne}$. The IR laser prepares $\mathrm{CN}-\mathrm{Ne}$ complexes with two quanta of $\mathrm{CN}$ stretch and possible intermolecular excitation. The UV laser subsequently promotes vibrationally excited $\mathrm{CN}-\mathrm{Ne}$ to the excited electronic state correlating with $\mathrm{CN} \mathrm{B}^{2} \Sigma^{+}(\mathrm{v}=0)+\mathrm{Ne}$, giving rise to a laser-induced fluorescence signal. Upon photolysis at $193 \mathrm{~nm}, \mathrm{CN}-\mathrm{Ne} X$ $\left(v_{\mathrm{CN}}=2\right)$ can also be observed directly in the jet expansion (without IR excitation $)$ and is probed on $B\left(v_{\mathrm{CN}}=0\right)-X\left(v_{\mathrm{CN}}=2\right)$ transitions, denoted as $\mathrm{UV}^{\prime}$. 
while UV enhancement spectra are obtained by fixing the IR laser on a $\mathrm{CN}-\mathrm{Ne}$ vibrational transition and scanning the UV laser.

Cyanogen iodide (ICN), which served as the $\mathrm{CN}$ radical precursor, is synthesized following methods described previously. ${ }^{21,22} \mathrm{ICN}$ vapor (1 torr at $25^{\circ} \mathrm{C}$ ) (Ref. 23) is entrained in a $75 \% \mathrm{Ne} / \mathrm{He}$ carrier gas (30 psi) by flowing the carrier gas over solid ICN contained in a polytetrafluoroethylene sample holder. ${ }^{9,11} \mathrm{CN}$ radicals are generated by $193 \mathrm{~nm}$ (Ref. 24) or $248 \mathrm{~nm}$ (Ref. 25) photolysis (Coherent, LPX 105i excimer laser) of the ICN vapor in the throat of a pulsed supersonic expansion (General Valve, Parker Hannifin Series 9). The photolytically generated $\mathrm{CN}$ radicals associate with $\mathrm{Ne}$ in the collisional region of the expansion to form $\mathrm{CN}-\mathrm{Ne}$ complexes. At a distance approximately 10 nozzle diameters downstream from the orifice, the gas mixture is interrogated with the spatially overlapped, counter-propagating IR and UV laser beams.

Tunable IR radiation with a $0.1 \mathrm{~cm}^{-1}$ bandwidth is generated with an optical parametric oscillator (OPO, LaserVision), pumped by an injection-seeded Nd:YAG laser (Continuum Precision II 8000), and operates in the $\mathrm{CN}$ overtone region at $\sim 2.5 \mu \mathrm{m}$ at typical pulse powers of $10 \mathrm{~mJ}$. The probe laser is the output of a Nd:YAG (Innolas, SL600) pumped dye laser (Radiant Dyes, Narrowscan) using Coumarin 460 dye, which operates in the vicinity of the $\mathrm{CN} B{ }^{2} \Sigma^{+}-X^{2} \Sigma^{+}(0,2)$ transition at $\sim 460 \mathrm{~nm}$ at considerably less than $1 \mathrm{~mJ} /$ pulse; the fundamental dye output has a bandwidth of $0.1 \mathrm{~cm}^{-1}$. The IR laser is focused into the center of the molecular beam using a $50 \mathrm{~cm}$ focal length lens, and is overlapped spatially with the collimated but unfocused UV laser. Both IR and UV lasers are calibrated using a wavelength meter (Coherent Wavemaster). The IR laser $(10 \mathrm{~Hz})$ is run at half the repetition rate of the UV laser $(20 \mathrm{~Hz})$ so that active background subtraction, [(IR + UV) - UV], can be employed on alternating laser shots to remove any background UV-only signal from IR-UV double resonance spectra. The delay between the IR and UV lasers is typically set at $50 \mathrm{~ns}$. The resulting fluorescence in the $\mathrm{CN}$ $B(\mathrm{v}=0)-X(\mathrm{v}=0)$ region (with a lifetime of $\tau=65 \mathrm{~ns})$ is collimated using $\mathrm{f} / 1$ optics and passed through a 390 (FWHM $=10 \mathrm{~nm}$ ) bandpass filter before impinging on a photomultiplier tube (ET Enterprises 9816B). The laser-induced fluorescence signal is then preamplified and displayed on a digital storage oscilloscope (LeCroy WaveRunner 44xi) attached to a PC for further analysis.

Rovibronic spectra of uncomplexed $\mathrm{CN}$ radicals were recorded to assess its rotational and vibrational distributions in the supersonic jet. The resulting experimental spectra were simulated using a rotational temperature of $\sim 4 \mathrm{~K}$, but with significant vibrational population observed in higher vibrational levels including $\mathrm{v}=2$ at both photolysis wavelengths. In addition, $\mathrm{CN}-\mathrm{Ne}$ complexes were readily detected with two quanta of $\mathrm{CN}$ vibrational excitation without IR excitation when $193 \mathrm{~nm}$ photolysis was utilized. In some cases, this resulted in a UV-only signal from the probe laser alone, which was subtracted in IR-UV double resonance spectra. This CN$\mathrm{Ne}$ UV-only signal was eliminated with photolysis at $248 \mathrm{~nm}$, resulting in nearly zero background when recording IR-UV spectra.
$A b$ initio potential energy surfaces were generated for $\mathrm{CN}$ $X^{2} \Sigma^{+}+\mathrm{Ne}$ and $\mathrm{CN} B^{2} \Sigma^{+}+\mathrm{Ne}$ as a function of the $\mathrm{CN}$ center-of-mass to Ne distance $(R)$ and intermolecular angle $(\theta)$ at a fixed, experimentally determined, $\mathrm{CN}$ bond length $(r=1.1718 \AA) .{ }^{26,27}$ The angle $\theta$ between $R$ and $r$ is defined as $0^{\circ}$ for the linear $\mathrm{CN}-\mathrm{Ne}$ configuration, adopting the convention of previous workers. The surfaces were generated using multireference configuration interaction (MRCI) energies with a Davidson correction (MRCI+Q), following the approach of Yang and Alexander ${ }^{15}$ and extending their prior work with extrapolation to the one electron complete basis set (CBS) limit. The procedures used for the MRCI calculations and extrapolation to the CBS limit are given in supplementary information. ${ }^{28}$ The potential energy landscape was generated by calculating energies every $0.25 \AA$ for $3 \AA<R<4.5 \AA$ and $R=6 \AA$ for 10 values of $\theta$ between $0^{\circ}(\mathrm{CN}-\mathrm{Ne})$ and $180^{\circ}(\mathrm{Ne}-$ $\mathrm{CN})$. All calculations were carried out in the MOLPRO suite of programs. ${ }^{29}$

\section{EXPERIMENTAL RESULTS}

Following $193 \mathrm{~nm}$ photolysis of ICN in Ne carrier gas, two new CN-Ne features are observed upon UV-only excitation in the $C N B(\mathrm{v}=0)-X(\mathrm{v}=2)$ spectral region, neither of which can be attributed to $\mathrm{CN}$ alone. Analogous $\mathrm{CN}-\mathrm{Ne}$ spectral features have been observed previously in the $\mathrm{CN} B$ $(\mathrm{v}=0)-X(\mathrm{v}=0)$ region by Heaven and co-workers. ${ }^{9,11}$ The $\mathrm{CN}-\mathrm{Ne}$ features in the $B(\mathrm{v}=0)-X(\mathrm{v}=2)$ region are assigned as transitions from $X\left(v_{\mathrm{CN}}=2, n^{K}=0^{0}\right)$ to $B\left(0,0^{0}\right.$ and $\left.1^{1}\right)$, with no intermolecular stretch $\left(v_{\mathrm{s}}\right)$ excitation, by comparison with the prior assignments in the $B(\mathrm{v}=0)-X(\mathrm{v}=0)$ region. The energy difference between analogous $\mathrm{CN}-\mathrm{Ne}$ features in the two regions is $4058.6(1) \mathrm{cm}^{-1}$ and corresponds to two quanta of $\mathrm{CN}$ stretch with no intermolecular excitation $\left(n^{K}=0^{0}\right)$ in the ground electronic state of the $\mathrm{CN}-\mathrm{Ne}$ complex. This value is within experimental uncertainty of the overtone transition of the $\mathrm{CN}$ monomer at $4058.55 \mathrm{~cm}^{-1}$. 30 The negligible change in the $\mathrm{CN}$ stretching frequency upon complexation is indicative of very little change in binding energy for $\mathrm{CN}-\mathrm{Ne}$ upon $\mathrm{CN}$ stretch excitation.

IR-UV double resonance spectra of $\mathrm{CN}-\mathrm{Ne}$ are also recorded with the IR laser preparing various hindered rotor states $\left(v_{\mathrm{CN}}=2, n^{K}\right)$ in the ground $X$ electronic state and subsequent UV laser excitation to $\left(v_{\mathrm{CN}}\right.$ $=0, n^{K}$ ) levels in the excited $B$ electronic state as shown in Fig. 1. IR-UV double resonance excitation was initially achieved with the IR laser positioned at $4061.7 \mathrm{~cm}^{-1}$. The UV laser was then scanned revealing five features in the UV enhancement spectra as shown in Fig. 2. Upper state assignments are made by comparing the energy and rotational band structures of the features observed in the $B(\mathrm{v}=0)-X(\mathrm{v}=2)$ region with those previously reported in the $B(\mathrm{v}=0)-X(\mathrm{v}=0)$ region (see Table I). The sum of the IR and $\mathrm{UV}$ transition frequencies correspond precisely to the UV transition frequencies of $\mathrm{CN}-\mathrm{Ne}$ features in the $B(\mathrm{v}=0)-X$ $(\mathrm{v}=0)$ region. ${ }^{9}$

IR spectra of $\mathrm{CN}-\mathrm{Ne}$, such as the band seen at $4061.7(1) \mathrm{cm}^{-1}$ in Fig. 3, are then recorded by scanning the IR laser with the UV laser fixed on the $Q$-branch of an 


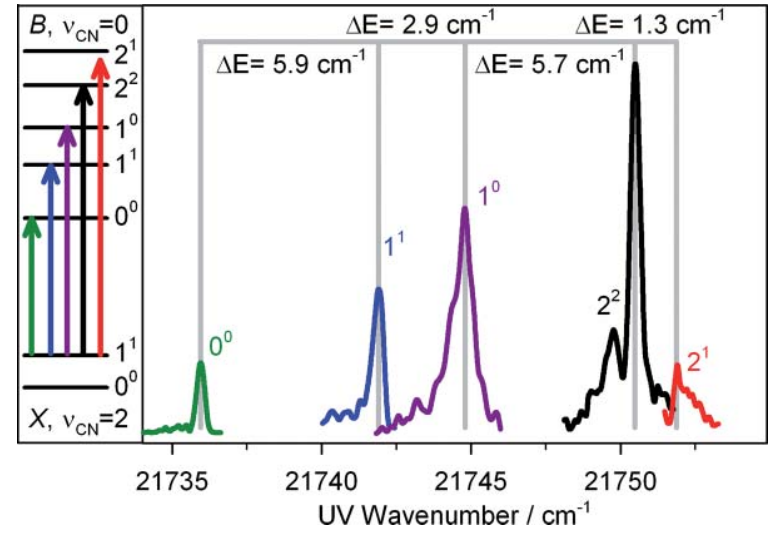

FIG. 2. UV transitions observed for $\mathrm{CN}-\mathrm{Ne}$ complexes in the $B\left(v_{\mathrm{CN}}=0\right)-X$ $\left(v_{\mathrm{CN}}=2\right)$ region following IR excitation at $4061.7 \mathrm{~cm}^{-1}$, which prepares the $X\left(v_{\mathrm{CN}}, n^{K}\right)=\left(2,1^{1}\right)$ state. The UV transitions terminate on various $n^{K}$ hindered rotor states (with $\Delta \mathrm{E}$ spacings) in the excited $B\left(v_{\mathrm{CN}}=0\right)$ electronic state as indicated by the energy level diagram and spectral band labels.

electronic transition, in this case at $21750.5(1) \mathrm{cm}^{-1}$. Positioning the UV laser on the $Q$-branch is advantageous for observing rotationally structured IR spectra without scanning the UV laser. Simulation of the UV transition indicates that the $Q$-branch encompasses $N \leq 7$ rotational levels as a result of power broadening, which adds a Lorentzian component to the linewidth of $0.5 \mathrm{~cm}^{-1}$. Spectra of other IR bands of $\mathrm{CN}-\mathrm{Ne}$ are recorded in a similar fashion, however, in each case using different UV transitions since the IR transitions terminate on different hindered rotor states. The band origins of the IR and UV transitions used to obtain IR-UV double-resonance spectra are listed in Table I. Varying the IR-UV time delay shows that the vibrationally activated $\mathrm{CN}-\mathrm{Ne}$ complexes are long lived, with lifetimes longer than several microseconds. The long lifetime of $\mathrm{CN}-\mathrm{Ne}$ upon $\mathrm{CN}$ overtone excitation is consistent with slow vibrational relaxation of $\mathrm{CN}(\mathrm{v}=2)$ with nonreactive partners studied previously. ${ }^{31,32}$

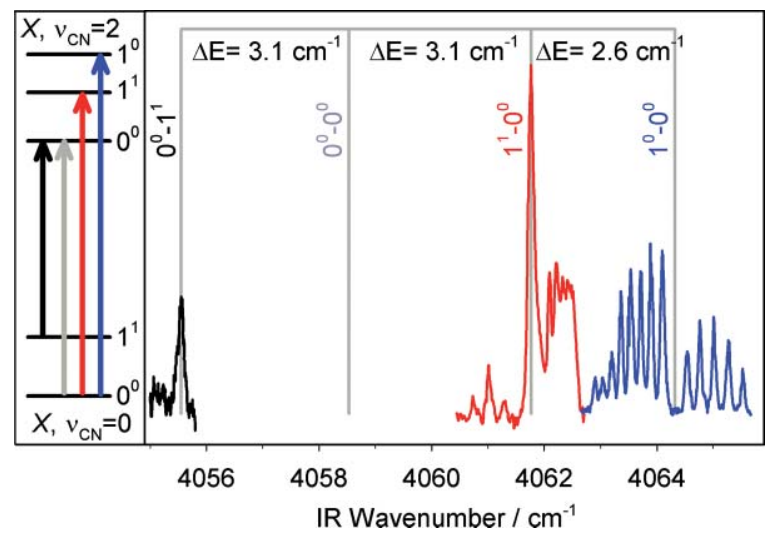

FIG. 3. Rotationally structured $\mathrm{CN}-\mathrm{Ne}$ bands in the $\mathrm{CN}$ overtone region observed by IR-UV double resonance spectroscopy. The $n^{K}$ hindered rotor states (and $\Delta \mathrm{E}$ spacings) associated with the IR transitions are indicated in the energy level diagram and spectroscopic labels. The $0^{0}-0^{0}$ transition (gray) is not observed in IR spectra because of $\Delta n= \pm 1$ selection rules, but is deduced from electronic transitions (see text and Table I).
TABLE I. Experimental CN-Ne band origins $\left(\mathrm{cm}^{-1}\right)$ with corresponding $\mathrm{CN}$ stretch and hindered rotor assignments $\left(v_{\mathrm{CN}}, n^{K}\right)$ for IR and UV transitions in IR-UV double resonance spectra and onephoton $B-X$ electronic transitions. Uncertainties in the band positions are consistent with the IR and UV laser bandwidths of $0.10 \mathrm{~cm}^{-1}$.

\begin{tabular}{lcc}
\hline \hline IR transition & UV transition & $B$ - $X$ transition $^{\mathrm{a}}$ \\
\hline $2045.6\left(1,1^{1}-0,0^{0}\right)$ & $23766.7\left(0,2^{2}-1,1^{1}\right)$ & $25812.3\left(0,2^{2}-0,0^{0}\right)$ \\
$4055.55\left(2,0^{0}-0,1^{1}\right)$ & $21745.0\left(0,1^{1}-2,0^{0}\right)$ & $25800.6\left(0,1^{1}-0,1^{1}\right)$ \\
{$\left[4058.6\left(2,0^{0}-0,0^{0}\right)\right]^{\mathrm{b}}$} & $21739.2\left(0,0^{0}-2,0^{0}\right)$ & $25797.8\left(0,0^{0}-0,0^{0}\right)$ \\
$4061.74\left(2,1^{1}-0,0^{0}\right)$ & $21736.0\left(0,0^{0}-2,1^{1}\right)$ & $25797.8\left(0,0^{0}-0,0^{0}\right)$ \\
& $21741.9\left(0,1^{1}-2,1^{1}\right)$ & $25803.7\left(0,1^{1}-0,0^{0}\right)$ \\
& $21744.8\left(0,1^{0}-2,1^{1}\right)$ & $25806.5\left(0,1^{0}-0,0^{0}\right)$ \\
& $21750.5\left(0,2^{2}-2,1^{1}\right)$ & $25812.3\left(0,2^{2}-0,0^{0}\right)$ \\
$4064.32\left(2,1^{0}-0,0^{0}\right)$ & $21751.9\left(0,2^{1}-2,1^{1}\right)$ & $25813.6\left(0,2^{1}-0,0^{0}\right)$ \\
$6051.6\left(3,1^{1}-0,0^{0}\right)$ & $21884.3\left(0,2^{1}-2,1^{0}\right)$ & $25813.6\left(0,2^{1}-0,0^{0}\right)$ \\
\hline \hline
\end{tabular}

${ }^{\mathrm{a}}$ Refs. 9 and 11.

${ }^{\mathrm{b}}$ This IR transition is not allowed and not observed directly in the present work. See text.

Figure 3 is a composite of the rotationally structured spectra for the three $\mathrm{CN}-\mathrm{Ne}$ bands observed in the $\mathrm{CN}$ overtone region. The position of the pure $\mathrm{CN}$ overtone stretch for $\mathrm{CN}-\mathrm{Ne},\left(v_{\mathrm{CN}}=2, n^{K}=0^{0}\right)-\left(0,0^{0}\right)$ at $4058.6 \mathrm{~cm}^{-1}$, is also shown but is derived from electronic spectra (above). The IR band at $4061.7 \mathrm{~cm}^{-1}$ is a perpendicular transition with characteristic $P, Q, R$ branch band structure, consistent with assignment as a $\Delta K=1$ transition. The transition at $4064.3 \mathrm{~cm}^{-1}$ is a parallel transition displaying $P$ and $R$ branches, but no $Q$ branch, indicative of a $\Delta K=0$ transition. Since the strongest infrared transitions are expected to originate from the ground state of CN-Ne $X\left(0,0^{\circ}\right)$ and follow a $\Delta n= \pm 1$ selection rule, as inferred from allowed $P$ - and $R$-lines in IR spectra of free $\mathrm{CN}$, the bands at 4061.7 and $4064.3 \mathrm{~cm}^{-1}$ are readily assigned to upper states with $\left(2,1^{1}\right)(\Delta K=1)$ and $\left(2,1^{0}\right)(\Delta K=0)$, respectively. The third feature observed at $4055.6(1) \mathrm{cm}^{-1}$ is attributed to a hot band since it lies to lower energy of the unobserved pure $\mathrm{CN}$ stretch of the complex. The $Q$-branch of the IR band is indicative of a $\Delta K=1$ transition, most likely due to the $\left(2,0^{0}\right)-\left(0,1^{1}\right)$ transition. This is validated by the sum of the IR and UV transition frequencies, $25800.6 \mathrm{~cm}^{-1}$, which has previously been attributed to a $B\left(v_{\mathrm{CN}}=0\right)-X\left(v_{\mathrm{CN}}=0\right)$ transition originating from the $\left(0,1^{1}\right)$ state. ${ }^{9}$

TABLE II. Spectroscopic constants $\left(\mathrm{cm}^{-1}\right)$ for $\mathrm{CN}-\mathrm{Ne}$ derived from infrared overtone spectra.

\begin{tabular}{|c|c|c|c|}
\hline & \multirow{2}{*}{$\frac{\text { Ground state }}{\left(0,0^{0}\right)}$} & \multicolumn{2}{|c|}{ Hindered internal rotor states } \\
\hline & & $\left(2,1^{1}\right)$ & $\left(2,1^{0}\right)$ \\
\hline$v_{0}$ (origin) & & $4061.74(10)$ & $4064.32(10)$ \\
\hline$B_{e}(\text { expt. })^{\mathrm{a}}$ & $0.106(2)$ & $0.093(2)$ & $0.111(2)$ \\
\hline$B$ (deperturbed) & & $0.108(2)$ & $0.097(2)$ \\
\hline$\left\langle R^{2}\right\rangle^{1 / 2}(\AA)$ & $3.70(4)$ & $3.71(4)$ & $3.92(4)$ \\
\hline$\beta\left[\left(2,1^{1}\right)-\left(2,1^{0}\right)\right]^{\mathrm{b}}$ & & & $0.195(4)$ \\
\hline
\end{tabular}

${ }^{a}$ Rotational constants from experimental data for $e$ symmetry levels.

${ }^{\mathrm{b}}$ Coriolis coupling term between $\left(\nu_{\mathrm{CN}}, n^{K}\right)=\left(2,1^{1}\right)$ and $\left(2,1^{0}\right)$ hindered rotor states. 
For a few experiments, the $\mathrm{OPO}$ is operated in the $\mathrm{CN}$ fundamental region at $4.9 \mu \mathrm{m}(0.5 \mathrm{~mJ} / \mathrm{pulse})$ and the second overtone region at $1.6 \mu \mathrm{m}(15 \mathrm{~mJ} / \mathrm{pulse})$. Strong IR transitions are observed for $\mathrm{CN}-\mathrm{Ne}$ at 2045.6(1) and 6051.6(1) $\mathrm{cm}^{-1}$, shifted 3.2(1) and 3.3(1) $\mathrm{cm}^{-1}$ to higher energy of the corresponding band origins for free $\mathrm{CN}$, respectively. For these experiments, the UV laser is positioned on $\mathrm{CN}-\mathrm{Ne} B\left(v_{\mathrm{CN}}\right.$ $=0)-X\left(v_{\mathrm{CN}}=1\right)$ and $B\left(v_{\mathrm{CN}}=1\right)-X\left(v_{\mathrm{CN}}=3\right)$ transitions at 23 766.7(1) and $21884.2(1) \mathrm{cm}^{-1}$, respectively. The sums of the IR and UV frequencies in these instances correspond to known CN-Ne $B\left(v_{\mathrm{CN}}=0\right)-X\left(v_{\mathrm{CN}}=0\right)$ and $B\left(v_{\mathrm{CN}}\right.$ $=1)-X\left(v_{\mathrm{CN}}=0\right)$ transitions, respectively. The similar shifts of the CN-Ne fundamental, overtone (see Fig. 3), and second overtone transitions from those in free $\mathrm{CN}$ leads to their assignment as $\left(v_{\mathrm{CN}}=1,2\right.$, or $\left.3,1^{1}\right)-\left(0,0^{0}\right)$ transitions. The similar shift in three spectral regions suggests that there is little change in the hindered rotor states with various degrees of $\mathrm{CN}$ vibrational excitation.

The rotational band structures of the IR features at $4061.7 \mathrm{~cm}^{-1}$ and $4064.3 \mathrm{~cm}^{-1}$ are not straightforward to analyze. The close proximity of the $n^{K}=1^{1}$ and $1^{0}$ upper states $\left(<3 \mathrm{~cm}^{-1}\right)$ will likely result in sizable Coriolis coupling or other perturbations that would lead to complicated rotational structures and intensity patterns. Initial rotational analyses of the $4061.7 \mathrm{~cm}^{-1}$ and $4064.3 \mathrm{~cm}^{-1}$ bands are carried out using the PGOPHER program for simulating rotational structure. ${ }^{33}$ The line positions of the $P$ and $R$ branches in both bands are fit by floating a common ground state $\left(0,0^{\circ}\right)$ rotational constant, $B_{00}$, the rotational constants for the two upper states $\left(2,1^{1}\right.$ and $\left.2,1^{0}\right), B_{11}$ and $B_{10}$, and band origins, $v_{11}$ and $v_{10}$, for both transitions. The unresolved $Q$-branch of the former transition is not included in the fit. The resultant spectral simulations with rotational temperature of $10 \mathrm{~K}$ and laser linewidth of $0.1 \mathrm{~cm}^{-1}$ are shown in Fig. 4 and the derived constants are listed in Table II.

Several aspects of the experimental and simulated rotational band structures shown in Fig. 4 are worthy of note. The $R$-branch of the $\left(2,1^{1}\right)-\left(0,0^{0}\right)$ band at $4061.7 \mathrm{~cm}^{-1}$ appears congested, indicative of an $R$-branch bandhead $\left(B_{11}\right.$ $<B_{00}$ ), suggesting that a perturbing state is pushing the rotational levels of the $\left(2,1^{1}\right)$ state downward. In addition, the position and shading of the $Q$-branch of the $\left(2,1^{1}\right)-\left(0,0^{0}\right)$ band is dramatically different from that predicted (dashed curve in upper panel) by spectroscopic constants (Table II) generated from analysis of the $\mathrm{P}$ and $\mathrm{R}$ branches. Furthermore, the relative intensities of $P$ and $R$ branch lines of the $\left(2,1^{0}\right)-\left(0,0^{0}\right)$ band at $4064.3 \mathrm{~cm}^{-1}$ do not match those anticipated from Hönl-London factors in the simulation (lower panel).

The preliminary rotational analysis of $P$ - and $R$-branch lines yields a ground state rotational constant, $B_{00}$, of $0.106(2) \mathrm{cm}^{-1}$, in good accord with that reported previously based on electronic spectroscopy studies. ${ }^{9,11}$ On the other hand, the derived upper state rotational constants, $B_{11}=0.093(2) \mathrm{cm}^{-1}$ and $B_{10}=0.111(2) \mathrm{cm}^{-1}$, differ significantly from the ground state value. The difference could arise from coupling between the two upper states and/or changes in the geometry of the complex as discussed below.
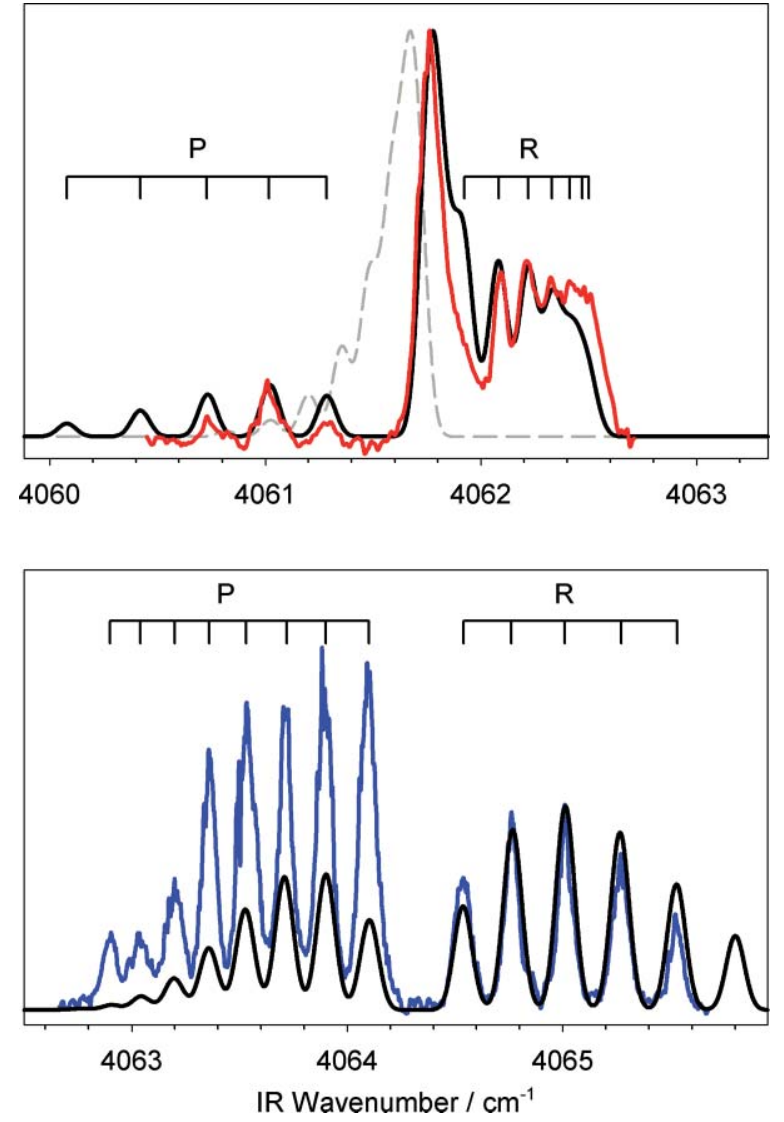

FIG. 4. Experimental and simulated rotational band contours for the $\left(v_{\mathrm{CN}}\right.$, $\left.n^{K}\right)=\left(2,1^{1}\right) \leftarrow\left(0,0^{0}\right)$ (upper panel) and $\left(2,1^{0}\right) \leftarrow\left(0,0^{0}\right)$ (lower panel) transitions. The red/blue traces are experimental spectra from IR-UV double resonance measurements, while the black traces are simulations with rotational temperature of $10 \mathrm{~K}$ and laser linewidth of $0.1 \mathrm{~cm}^{-1}$. The simulations are based on fits to $P$ - and $R$-branch lines only. Rotational assignments are shown as ticks. The dashed gray line illustrates the contour for the $Q$-branch anticipated using the same (perturbed) rotational constant for the $\left(2,1^{1}\right)$ upper state. The experimental $Q$-branch structure is well represented (red) using the deperturbed rotational constant derived for the $\left(2,1^{1}\right)$ upper state. The simulated line intensities are based on Hönl-London factors.

\section{ANALYSIS}

\section{A. Coriolis coupling effects and deperturbation analysis}

As presented in Sec. III, several aspects of the rotationally structured $\mathrm{CN}-\mathrm{Ne}$ bands suggest a perturbation between the $\left(\mathrm{v}_{\mathrm{CN}}, n^{K}\right)=\left(2,1^{1}\right)$ and $\left(2,1^{0}\right)$ upper states. A significant body of work has been published on infrared spectra of closed-shell HX and DX ( $\mathrm{X}=\mathrm{F}, \mathrm{Cl})$-Rg complexes in which Coriolis coupling between closely spaced states has been used to explain very similar experimental findings. ${ }^{34-36}$ These HXRg systems exhibit $J$-dependent shifts in energy levels and deviations in rotational transition strengths, which were analyzed as an $l$-doubling perturbation between intermolecular bending and/or stretching levels. A similar analysis is performed here for $\mathrm{CN}-\mathrm{Ne}$ to elucidate "deperturbed" rotational constants for the bands in question, and to account for the line positions and band shapes observed. However, no attempt is made to explicitly treat the line intensities, which are influenced by both Coriolis coupling and the double resonance nature of the experiments. 
The HX-Rg systems studied by Nesbitt and co-workers were treated as linear complexes, in which the lowest $\Sigma-\Sigma$ bending transition terminates on $e$ symmetry upper rotational levels (with equivalent notation of $n^{K}=1^{0}$ for the upper state); this is also the case for $P$ - and $R$-branch lines of the $\Pi$ $\Sigma$ band (with $n^{K}=1^{1}$ upper state). The $Q$-branch lines of the $\Pi-\Sigma$ bending transition, however, terminate on $f$ symmetry upper levels. The close proximity of the two upper states can give rise to noticeable Coriolis coupling between $e$ symmetry rotational levels, which is treated by modeling the upper state energy levels through a modified rotational Hamiltonian. The $Q$-branch of the $\Pi-\Sigma$ transition is not perturbed.

Analogous Coriolis coupling is immediately evident in $\mathrm{CN}-\mathrm{Ne}$ overtone spectra. Preliminary analysis of the transition to the $\left(2,1^{1}\right)$ state indicates that the upper state rotational constant for $e$ symmetry levels, $B_{11}$, derived from $P$ and $R$ branch lines, is reduced relative to the unperturbed ground state value. The $Q$ branch of this transition, which terminates solely on $f$ symmetry levels, is not resolved and therefore cannot be used to obtain an unperturbed rotational constant for the $\left(2,1^{1}\right)$ state; thus, we utilize $B_{00}$ for comparisons. Assuming the reduction in rotational constant for the $1^{1}$ state is a result of Coriolis coupling, we deduce that the perturbing state lies higher in energy and is presumably the nearby $\left(2,1^{0}\right)$ state.

A complete deperturbation analysis was then undertaken using a modified rotational Hamiltonian

$$
\hat{H}=\left(\begin{array}{ll}
H_{11} & H_{12} \\
H_{21} & H_{22}
\end{array}\right),
$$

with matrix elements

$$
\begin{aligned}
& H_{11}=v_{10}+B_{10}\left[N(N+1)-K^{2}\right], \\
& H_{22}=v_{11}+B_{11}\left[N(N+1)-K^{2}\right], \\
& H_{12}=H_{21}=\beta[N(N+1)]^{1 / 2},
\end{aligned}
$$

where $B_{10}$ and $B_{11}$ are the deperturbed rotational constants for the $\left(2,1^{0}\right)$ and $\left(2,1^{1}\right)$ states, $v_{10}$ and $v_{11}$ are their respective band origins listed in Table II. The constant $\beta$ is related to the magnitude of the Coriolis coupling. The eigenvalues for this $N$-dependent Hamiltonian are fit using a leastsquares method to rotational energy levels for the $\left(2,1^{0}\right)$ and $\left(2,1^{1}\right)$ states, which were obtained from simulation of $P$ and $R$ branch lines described in Sec. III. All parameters except the band origins are allowed to float in the fitting procedure. Fitting the rotational energy levels in this fashion reveals a strong Coriolis coupling between the $\left(2,1^{0}\right)$ and $\left(2,1^{1}\right)$ hindered internal rotor states of $\mathrm{CN}-\mathrm{Ne}$, which are separated by only $2.6(1) \mathrm{cm}^{-1}$, with $\beta$ of $0.195 \mathrm{~cm}^{-1}$ and deperturbed rotational constants also listed in Table II. The deperturbation analysis shows that Coriolis coupling increases / decreases the magnitude of the experimentally observed rotational constant for the $\left(2,1^{0}\right) /\left(2,1^{1}\right)(f$ symmetry) state by nearly $20 \%$ compared to its unperturbed value.

The rotational constants for the ground state and hindered internal rotor states, the latter following deperturbation, are then used to evaluate the average intermolecular distance, $\left\langle R^{2}\right\rangle^{1 / 2}$, between the center-of-mass of $\mathrm{CN}$ and Ne using a pseudo-diatomic model. This yields an average separation distance in the ground $\left(0,0^{0}\right)$ state of 3.70(4) $\AA$. The average separation in the vibrationally excited $\left(2,1^{1}\right)$ state is essentially unchanged at 3.71(4) $\AA$, whereas the $\left(2,1^{0}\right)$ state shows a substantial increase in the average distance of 3.92(4) $\AA$. This indicates a significant change in the properties of hindered rotor states with $n^{K}=1^{1}$ and $1^{0}$ as discussed below.

\section{B. Hindered rotor analysis}

The IR-UV double resonance experiments reveal several hindered rotor states supported by the $\mathrm{CN} X^{2} \Sigma^{+}(\mathrm{v}=2)$ + Ne intermolecular potential, namely states with $n^{K}=0^{0}$, $1^{1}$, and $1^{0}$. No intermolecular stretching states have been observed and thus we focus our theoretical analysis exclusively on the angular states. In this section, we use the assignments and energy spacings of these hindered internal rotor states to deduce information about the anisotropy of the $\mathrm{CN} X^{2} \Sigma^{+}$ $(\mathrm{v}=2)+\mathrm{Ne}$ potential based solely on the experimental results, which is then extended to $\mathrm{CN} X^{2} \Sigma^{+}(\mathrm{v}=0)+\mathrm{Ne}$ and compared with prior results on $\mathrm{CN} B^{2} \Sigma^{+}(\mathrm{v}=0)+\mathrm{Ne} .{ }^{9}$ The resultant angular potentials for $\mathrm{CN}\left(X^{2} \Sigma^{+}, B^{2} \Sigma^{+}\right)+\mathrm{Ne}$ are then compared with those obtained from high level $a b$ initio theory in Sec. V.

The hindered rotor analysis is based on the theoretical treatment for open-shell diatom-rare gas complexes developed by Dubernet et al. ${ }^{18}$ (and briefly summarized in supplementary information ${ }^{28}$ ), utilizing two parameters, $V_{10}$ and $V_{20}$, to describe the anisotropy of the system. The parameters can be determined directly from the hindered rotor state spacings observed experimentally, and can then be inserted into the truncated Legendre polynomial

$$
V(\theta)=V_{10} \cos (\theta)+\frac{V_{20}\left(3 \cos ^{2}(\theta)-1\right)}{2},
$$

in order to model the angular intermolecular potential while neglecting any isotropic contribution.

Dubernet et $a l .{ }^{18}$ provide a discussion of the limits imposed on these parameters; however, certain restrictions can be derived from the experimental data alone. For example, a simple inspection of the $\mathrm{CN}-\mathrm{Ne} X\left(v_{\mathrm{CN}}=2\right)$ hindered rotor energy level spacings shows that $V_{10}$ is closer to the limit of $V_{10}=0$ than $V_{10}=2 b$ (illustrated by Fig. 2 of Ref. 18). Furthermore, the energy ordering of the $\left(2,1^{1}\right)$ and $\left(2,1^{0}\right)$ states indicates that $V_{20}$ is positive, while the larger spacing between the $\left(2,0^{0}\right)$ and $\left(2,1^{1}\right)$ states as compared to the $\left(2,1^{1}\right)$ and $\left(2,1^{0}\right)$ states limits the magnitude of $V_{20}$ to less than $5.1 \mathrm{~cm}^{-1}$. A unique solution for $V_{10}$ and $V_{20}$ is found within these constraints yielding values of $-1.6 \mathrm{~cm}^{-1}$ and $4.3 \mathrm{~cm}^{-1}$, respectively, which reproduce the experimentally observed energy spacings precisely. To account for the experimental uncertainty associated with measurement of the IR band origins $\left( \pm 0.1 \mathrm{~cm}^{-1}\right)$, we also derive a corresponding range of $V_{10}$ and $V_{20}$ values, specifically -2.1 to $-0.8 \mathrm{~cm}^{-1}$ for $V_{10}$ and 4.3 $\pm 0.1 \mathrm{~cm}^{-1}$ for $V_{20}$. It should be noted that sign of $V_{10}$, indicating a preference for $\mathrm{Ne}$ binding to a specific end of the $\mathrm{CN}$ radical, cannot be determined from the experimental methods or associated theoretical analysis used in this work and as 
such the sign of $V_{10}$ is arbitrary; we assume $V_{10}$ is negative following prior work.

The corresponding energy spacings for $\mathrm{CN}-\mathrm{Ne} X\left(v_{\mathrm{CN}}\right.$ $=0)$ were then calculated using the same values for the potential anisotropy parameters $\left(V_{10}=-1.6 \mathrm{~cm}^{-1}, V_{20}\right.$ $=4.3 \mathrm{~cm}^{-1}$ ) and the appropriate monomer rotational constant for $\mathrm{CN}^{2} \Sigma^{+}(\mathrm{v}=0)$. This yields an energy spacing between $\mathrm{CN}-\mathrm{Ne} X\left(0,0^{0}\right)$ and $\left(0,1^{1}\right)$ states, which is in excellent agreement with the only experimentally observed spacing of 3.1(1) $\mathrm{cm}^{-1}$. These anisotropy parameters for the $X\left(v_{\mathrm{CN}}=0\right)$ state of $\mathrm{CN}-\mathrm{Ne}$ differ somewhat from those reported previously, particularly for $V_{20}{ }^{9}$

Repeating this analysis for $\mathrm{CN}-\mathrm{Ne} B\left(v_{\mathrm{CN}}=0\right)$, based on the $n^{K}=0^{0}, 1^{1}, 1^{0}, 2^{2}$, and $2^{1}$ hindered rotor states observed in the $B(\mathrm{v}=0)-X(\mathrm{v}=2)$ and $B(\mathrm{v}=0)-X(\mathrm{v}=0)$ spectral regions, we obtain values of $8.2 \mathrm{~cm}^{-1}$ and $0.6 \mathrm{~cm}^{-1}$ for the potential anisotropy parameters $V_{10}$ and $V_{20}$, respectively. These parameters are in good accord with results from a prior analysis by Fei and Heaven. ${ }^{9}$

The effective angular dependence of the $\mathrm{CN} X^{2} \Sigma^{+}$(v) $+\mathrm{Ne}$ potential is then plotted using the truncated Legendre polynomial expansion shown in Eq. (5). The $V_{10}$ and $V_{20}$ anisotropy parameters are radially averaged over the intermolecular distances sampled experimentally for $\mathrm{CN} X$ $(\mathrm{v}=2)$ and are consistent with the data available for CN $X$ $(\mathrm{v}=0)$, where rotational analysis indicates an average $\mathrm{CN}-\mathrm{Ne}$ separation of $\left\langle R^{2}\right\rangle^{1 / 2}=3.70(4) \AA$ for the ground state. $V(\theta)$ as well as the individual terms in Eq. (5) with associated uncertainties (shown as shaded regions) are plotted in the upper panel of Fig. 5. A similar graph shown in the upper panel of Fig. 6 depicts the effective angular dependence of the $\mathrm{CN} B$ ${ }^{2} \Sigma^{+}(\mathrm{v}=0)+$ Ne potential for comparison, again neglecting isotropic contributions. The angular dependence of the potentials derived from this analysis of hindered internal rotor states of the $\mathrm{CN}-\mathrm{Ne}$ complex are discussed in the following section.

\section{DISCUSSION}

\section{A. Experimental determination of potential anisotropy}

The angular potential $V(\theta)$ for $\mathrm{CN} X^{2} \Sigma^{+}+\mathrm{Ne}$, derived from the analysis of observed hindered rotor states (Sec. IV and supplementary information ${ }^{28}$ ) and shown in the upper panel of Fig. 5, indicates that the minimum energy geometry of $\mathrm{CN} X^{2} \Sigma^{+}+\mathrm{Ne}$ is nonlinear with a shallow minimum at $\theta \sim 80^{\circ}$. The ground state angular potential is dominated by the $V_{20}$ anisotropy term, although the smaller $V_{10}$ term tips the minimum away from $90^{\circ}$. The barrier $\left(\theta \sim 0^{\circ}, 180^{\circ}\right)$ to internal $\mathrm{CN}$ rotation is only $\sim 8 \mathrm{~cm}^{-1}$, indicating a weakly anisotropic potential close to the free rotor limit. As a result, the ground state of the $\mathrm{CN}-\mathrm{Ne}$ complex will have a bent configuration on average, while undergoing wide amplitude motion in the internal bending coordinate. By contrast, the $\mathrm{CN}$ $B^{2} \Sigma^{+}+$Ne potential, plotted in the upper panel of Fig. 6, shows that the minimum energy configuration in the excited $B$ electronic state is linear with a somewhat higher barrier $(\theta$ $\sim 180^{\circ}$ ) to internal rotation of $\sim 16 \mathrm{~cm}^{-1}$. In this case, the potential anisotropy is dominated by the $V_{10}$ term.

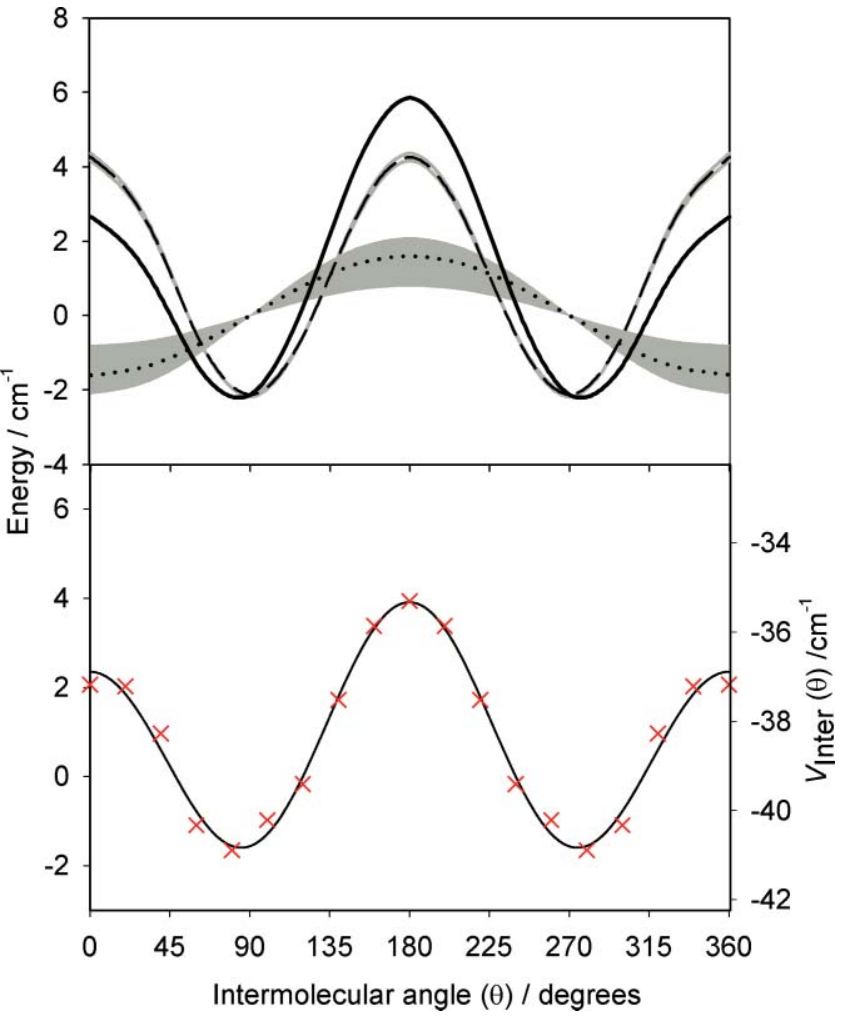

FIG. 5. Potential anisotropy for $\mathrm{CN} X^{2} \Sigma^{+}\left(v_{\mathrm{CN}}=2\right)+\mathrm{Ne}$ as a function of intermolecular angle $\theta$. Upper panel: The solid line indicates the effective angular potential, represented by Eq. (5), while the dotted and dashed lines give the individual contributions from the smaller $V_{10}$ and dominant $V_{20}$ terms derived from the experimental hindered rotor spacings. The gray shaded regions indicate the experimental uncertainties associated with $V_{10}$ and $V_{20}$. Lower panel: The ab initio data points are illustrated by red crosses. The black trace is a fit to the ab initio data using the Legendre expansion with an additional isotropic component.

The anisotropy parameters and correlation diagram shown in supplementary Fig. $\mathrm{S}^{28}$ were generated using the $\mathrm{CN} X(\mathrm{v}=2)$ monomer rotational constant. Regeneration of the correlation diagram for $\mathrm{CN} X^{2} \Sigma^{+}(\mathrm{v}=0,1$, and 3$)+\mathrm{Ne}$, using the appropriate $\mathrm{CN}$ monomer rotational constants with identical $V_{10}$ and $V_{20}$ anisotropy parameters, predicts $0^{0}$ to $1^{1}$ hindered rotor spacings in each $\mathrm{CN}$ spectral region. The values produced in this fashion are consistent with the spacing in each region between the experimentally observed $\mathrm{CN}-\mathrm{Ne}$ $1^{1}$ feature and the anticipated position of the $0^{0}$ band, which is assumed to be unchanged from the $\mathrm{CN}$ monomer stretch. Thus, the potential anisotropy for $\mathrm{CN} X^{2} \Sigma^{+}+\mathrm{Ne}$ shown in Fig. 5 appears to be essentially unchanged for $\mathrm{CN}(\mathrm{v}=0-4)$.

\section{B. $A b$ initio $\mathrm{MRCl}$ potentials for $\mathrm{CN}\left(X^{2} \Sigma^{+}, B^{2} \Sigma^{+}\right)+\mathrm{Ne}$}

Figure 7 shows 2D ab initio potential energy surfaces (PES) for the ground $\mathrm{CN} X^{2} \Sigma^{+}+\mathrm{Ne}$ and excited $\mathrm{CN} B^{2} \Sigma^{+}$ $+\mathrm{Ne}$ electronic states, generated in the fashion described in Sec. II and supplementary information. ${ }^{28}$ The ground state surface obtained using the MRCI+Q/CBS method builds on that previously reported by Yang and Alexander ${ }^{15}$ based on MRCI+Q/aVTZ calculations with both counterpoise and size-consistency corrections. The ab initio PES generated in 


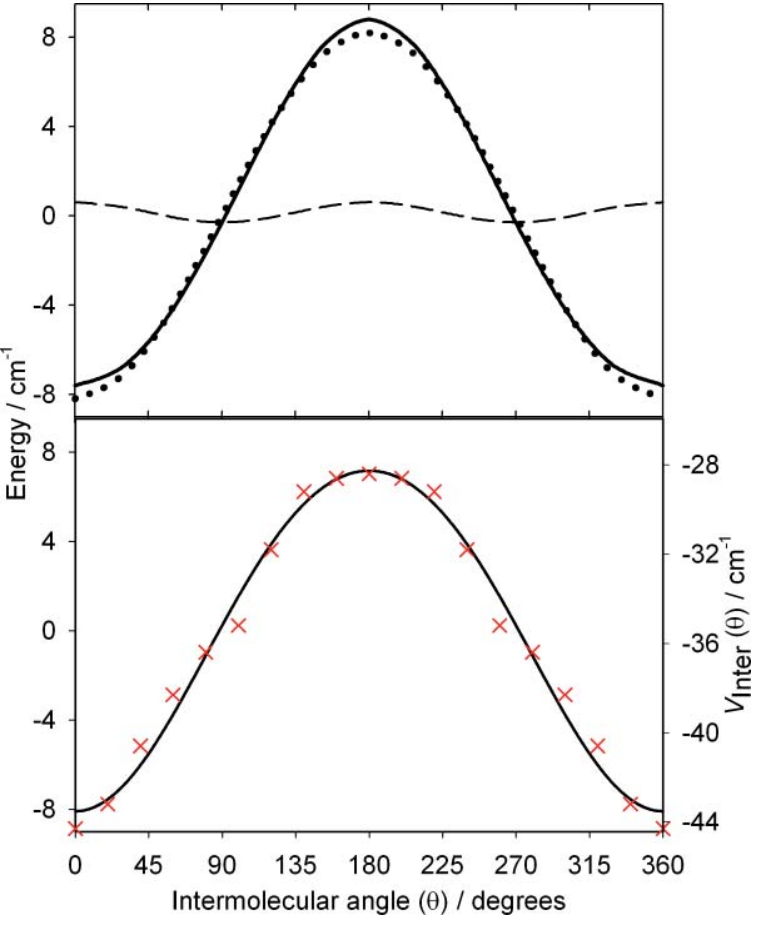

FIG. 6. Potential anisotropy for $\mathrm{CN} B^{2} \Sigma^{+}\left(v_{\mathrm{CN}}=0\right)+\mathrm{Ne}$ as a function of $\theta$. Upper panel: The solid line indicates the effective angular potential, represented by Eq. (5), while the dotted and dashed lines represent the dominant $V_{10}$ and much smaller $V_{20}$ terms deduced from the experimental hindered rotor spacings in the excited electronic state. Lower panel: The $a b$ initio data points are indicated by red crosses. The black trace is a fit to the ab initio data using the Legendre expansion with an additional isotropic component.

this work exhibits a shallow global minimum at $\theta=80^{\circ}$, consistent with the experimentally determined anisotropy for the $\mathrm{CN} X^{2} \Sigma^{+}+$Ne potential, but differs from the nearly isotropic potential with very shallow minima at linear $\theta$ $=0^{\circ}$ and $180^{\circ}$ configurations predicted using an AVTZ basis set. ${ }^{15}$ Fitting a radial slice of the potential landscape to a Morse potential at the minimum energy geometry $\left(\theta=80^{\circ}\right)$ yields an intermolecular distance of $R_{\mathrm{e}}=3.51 \AA$ and a dissociation energy of $D_{\mathrm{e}} \sim 41 \mathrm{~cm}^{-1}$.

The lower panel of Fig. 5 shows the angular dependence of the $a b$ initio potential for $\mathrm{CN} X^{2} \Sigma^{+}+\mathrm{Ne}$. The theoretical curve was generated by fitting the radial coordinate at fixed values of $\theta$ to a Morse potential, and thereby obtaining the minimum energy at each $\theta$. Combining the results at each angle produces a $1 \mathrm{D}$ minimum energy path through the $2 \mathrm{D}$ intermolecular potential. This angular potential was fit to the Legendre expansion shown in Eq. (5) in order to evaluate theoretical anisotropy parameters, yielding $V_{10}$ and $V_{20}$ values of $-0.8 \pm 0.1 \mathrm{~cm}^{-1}$ and $3.2 \pm 0.2 \mathrm{~cm}^{-1}$, respectively, for CN $X$ ${ }^{2} \Sigma^{+}+\mathrm{Ne}$, where the uncertainty represents $1 \sigma$ in the Legendre fitting procedure.

The ab initio $\mathrm{CN} B^{2} \Sigma^{+}+\mathrm{Ne}$ PES (upper panel of Fig. 7) exhibits a much higher degree of anisotropy than the ground state, favoring a linear geometry $\left(\theta=0^{\circ}\right)$ with $R_{\mathrm{e}}=3.76 \AA$ and $D_{\mathrm{e}} \sim 44 \mathrm{~cm}^{-1}$ evaluated in the same manner as above. Once again, a minimum energy angular path through the potential energy landscape was generated and fit to a truncated Legendre polynomial, shown in the lower panel of Fig. 6. The
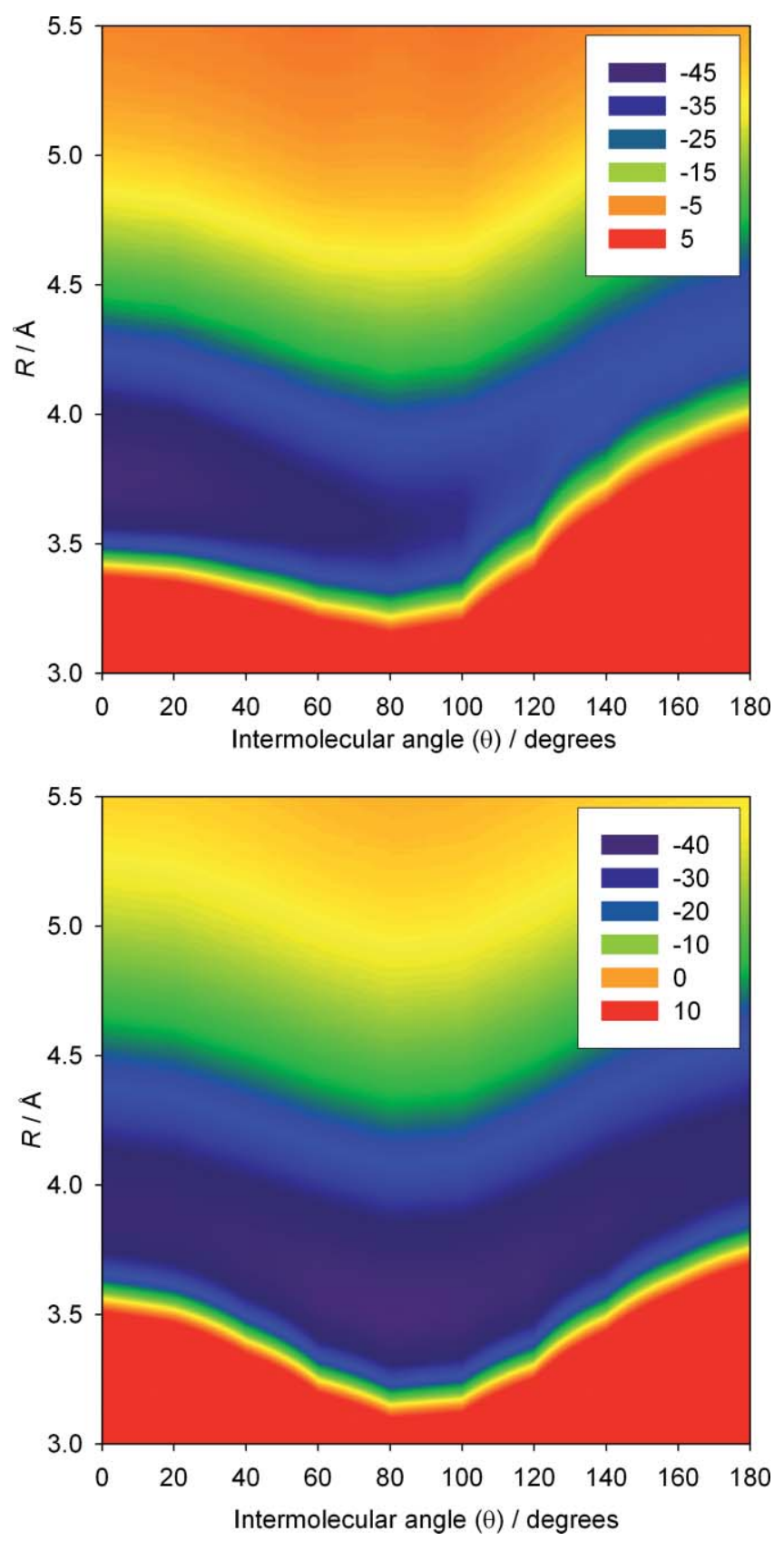

FIG. 7. CASSCF(9,8)//MRCI+Q/CBS ab initio potentials $\left(\mathrm{cm}^{-1}\right)$ for the $\mathrm{CN} X{ }^{2} \Sigma^{+}+\mathrm{Ne}$ and $\mathrm{CN} B{ }^{2} \Sigma^{+}+\mathrm{Ne}$ electronic states. Points were generated every $0.25 \AA$ in the radial coordinate between 3 and $4.5 \AA$ and then at $6 \AA$. Radial data were produced every $20^{\circ}$ between $\theta=0^{\circ}$ and $180^{\circ}$. The potential energy surfaces show distinctly different minimum energy geometries. Lower panel: $\mathrm{CN} X^{2} \Sigma^{+}$ $+\mathrm{Ne}$ displays a potential minimum at $\theta=80^{\circ}$ with an $R_{\mathrm{e}}$ of $\sim 3.5 \AA$ and a $D_{\mathrm{e}}$ of $41 \mathrm{~cm}^{-1}$. Upper panel: $\mathrm{CN} B{ }^{2} \Sigma^{+}+$Ne exhibits a potential minimum for linear CN-Ne $\left(\theta=0^{\circ}\right)$ with a significantly longer $R_{\mathrm{e}}$ of $\sim 3.75 \AA$ and a $D_{\mathrm{e}}$ of $44 \mathrm{~cm}^{-1}$.

$\mathrm{CN} B{ }^{2} \Sigma^{+}+$Ne potential anisotropy is dominated by a $V_{10}$ term of $-7.6 \pm 0.3 \mathrm{~cm}^{-1}$, with significantly less dependence on $V_{20}\left(-0.5 \pm 0.4 \mathrm{~cm}^{-1}\right)$.

Figures 5 and 6 allow comparisons between the theoretical anisotropy parameters and those determined experimentally. The $a b$ initio calculations reproduce the angular dependence of the $\mathrm{CN} X{ }^{2} \Sigma^{+}+\mathrm{Ne}$ and $\mathrm{CN} B$ ${ }^{2} \Sigma^{+}+$Ne potentials remarkably well. The relative signs of 
the $V_{10}$ anisotropy parameters can also be determined from these $a b$ initio potentials. The excellent agreement between experiment and theory validates these new $a b$ initio potentials and the need for complete basis set extrapolation, rather than utilizing counterpoise correction and basis set superposition error approximations with small basis sets.

\section{Generation of body fixed wavefunctions and implications}

Wavefunctions for the hindered rotor state are produced through the diagonalization of the experimental angular potential for $\mathrm{CN} X^{2} \Sigma^{+}+\mathrm{Ne}$ (Fig. 5). As in previous studies of HX complexes, ${ }^{36,37}$ the potential is diagonalized in a bodyfixed free rotor basis to give the angular eigenfunctions as a function of $\theta$. The probability distributions for the $n^{K}=0^{0}$, $1^{1}$ and $1^{0}$ levels are shown in Fig. 8. In each case, the broad distributions are indicative of wide amplitude hindered internal rotor motion. The probability distribution for the $0^{0}$ level is peaked in bent configurations slightly favoring the $\mathrm{N}$-side of $\mathrm{CN}$, whereas free rotor behavior samples all configurations with equal probability. The $1^{0}$ level has maximum probability at linear $\mathrm{Ne}-\mathrm{CN}$ and $\mathrm{CN}-\mathrm{Ne}$ orientations, with greater integrated likelihood for $\mathrm{Ne}$ to be located on the $\mathrm{N}$-side of $\mathrm{CN}$ compared to free rotor behavior. The $1^{1}$ level is nearly free rotor-like with a most probable bent configuration.

The probability distributions can also be used to explain the different rotational constants (deperturbed) obtained for the $\mathrm{CN}-\mathrm{Ne}\left(2,1^{0}\right)$ hindered rotor state as compared to the $\left(2,1^{1}\right)$ and $\left(0,0^{0}\right)$ levels (see Table II). The rotational constant for the $\mathrm{CN}-\mathrm{Ne}\left(2,1^{0}\right)$ level is some $10 \%$ smaller than those for the other two levels, indicating a corresponding increase in the $\mathrm{Ne}$ to $\mathrm{CN}$ (center-of-mass) distance for the $\mathrm{CN}-\mathrm{Ne}\left(2,1^{0}\right)$ level. The longer effective bond length for the $1^{0}$ level appears to stem from its most probable linear configuration, which differs from the $0^{0}$ and $1^{1}$ levels where nonlinear configurations are most probable. This can be understood by examining the $\mathrm{CN} X^{2} \Sigma^{+}+\mathrm{Ne} a b$ initio potential (Fig. 7), which shows that the optimized intermolecular bond length changes from $3.5 \AA$ at the global minimum with $\theta=80^{\circ}$ to $3.9 \AA$ for linear $\mathrm{CN}-\mathrm{Ne}$ $\left(\theta=0^{\circ}\right)$. The van der Waals interaction allows the $\mathrm{Ne}$ atom to get closer to $\mathrm{C} \equiv \mathrm{N}$ center-of-mass on a sideways approach as compared to a head-on approach to either end of the $\mathrm{CN}$ radical. The experimentally derived average bond lengths follow this trend with 3.71(4) $\AA$ for the $\left(2,1^{1}\right)$ level and 3.70(4) $\AA$ for the $\left(0,0^{0}\right)$ level, which have bent configurations, and 3.92(4) $\AA$ for the $\left(2,1^{0}\right)$ level with a linear most probable configuration.

\section{Comparison with previous work}

Rotational analysis of the IR-UV double resonance spectra reported in this work has afforded the re-evaluation of the $\mathrm{CN}-\mathrm{Ne}$ ground state rotational constant as $0.106(1) \mathrm{cm}^{-1}$, which is in excellent agreement with the value previously reported by Fei and Heaven..$^{9}$ The weak coupling of the $\mathrm{CN}$ stretch to intermolecular degrees of freedom is evident in the very small shift in $\mathrm{CN}$ stretching frequency upon com-

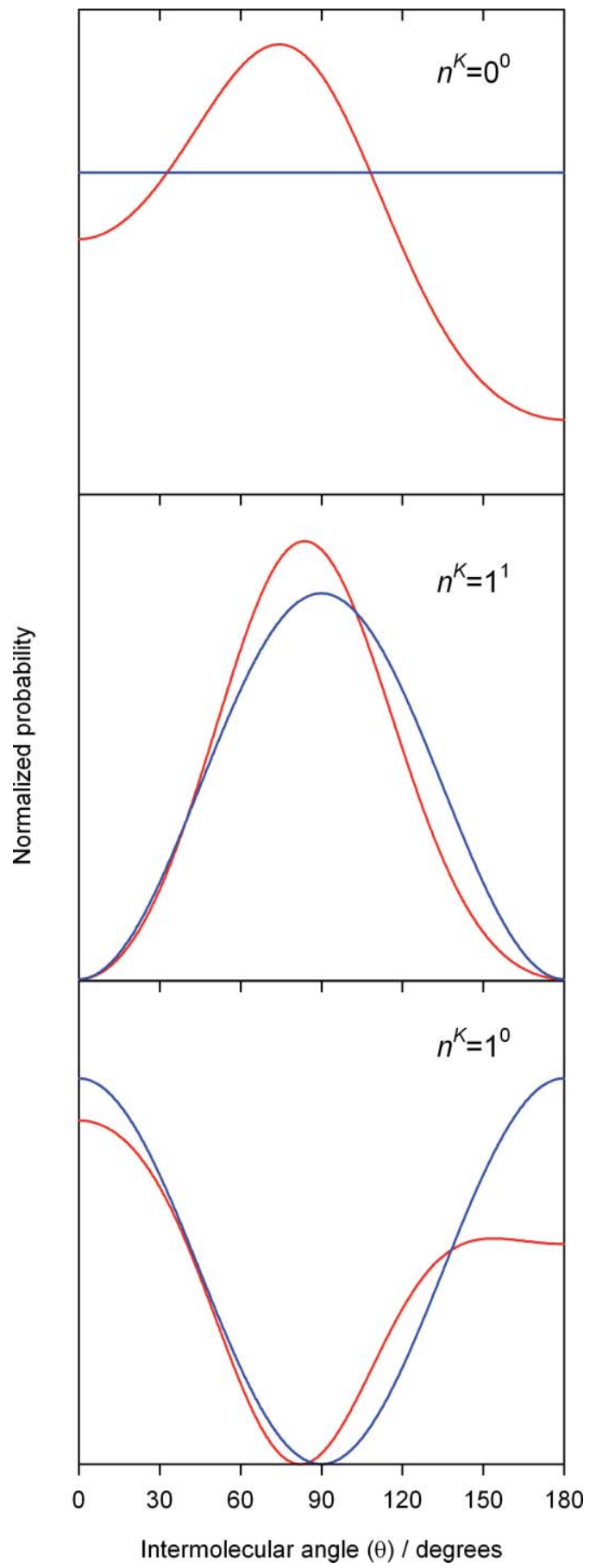

FIG. 8. Hindered rotor probability distributions for the $\mathrm{CN}-\mathrm{Ne}\left(0,0^{0}\right)$, CN$\mathrm{Ne}\left(0,1^{0}\right)$, and $\mathrm{CN}-\mathrm{Ne}\left(0,1^{1}\right)$ states (red) correlating with $\mathrm{CN} X^{2} \Sigma^{+}+\mathrm{Ne}$ compared with the free rotor probability distributions (blue). The hindered rotor wavefunctions are generated from the body-fixed diagonalization of the experimental angular potential (Fig. 5).

plexation, which was also noted in the previous electronic spectroscopy. The IR spectra obtained in the fundamental, overtone, and second overtone regions (for analogous hindered rotor states) show that the $\mathrm{CN}-\mathrm{Ne}$ hindered rotor band positions change very little with increasing quanta of vibration in the $\mathrm{C}-\mathrm{N}$ stretching coordinate as compared with free $\mathrm{CN}$ stretch. The experimental spectra have also allowed the assignment of accurate anisotropy parameters for $\mathrm{CN}$ 
$X^{2} \Sigma^{+}+\mathrm{Ne}$, where $V_{10}$ and $V_{20}$ are $-1.6 \mathrm{~cm}^{-1}$ and 4.3 $\mathrm{cm}^{-1}$, respectively, and for $\mathrm{CN} B^{2} \Sigma^{+}+$Ne where $V_{10}$ and $V_{20}$ are $-8.2 \mathrm{~cm}^{-1}$ and $0.6 \mathrm{~cm}^{-1}$. These values have been determined directly from the energy spacings of hindered internal rotor states, with $V_{20}$ for $\mathrm{CN} X^{2} \Sigma^{+}+\mathrm{Ne}$ close to the value estimated $\left(5.1 \mathrm{~cm}^{-1}\right),{ }^{9}$ and with the excited state values in excellent agreement with those reported by Lin and Heaven. ${ }^{11}$

The new ab initio potential energy surface for $\mathrm{CN} X^{2} \Sigma^{+}$ $+\mathrm{Ne}$ shows the weakly anisotropic ground state potential energy surface to have a nonlinear minimum energy configuration, consistent with the bent minimum energy configuration predicted with MP2 methods, ${ }^{16}$ yet contrary to the linear minimum energy geometry for the nearly isotropic potential reported by Yang and Alexander. ${ }^{15}$ The new $\mathrm{CN} B^{2} \Sigma^{+}+\mathrm{Ne}$ potential indicates that the electronically excited state favors a linear configuration. These potentials have been used to produce minimum energy paths as a function of intermolecular angle for both ground and electronically excited states, and these are in very good agreement with those generated from the experimental hindered rotor analysis. These surfaces have also been used to determine the signs of the anisotropy parameters.

\section{CONCLUSIONS}

The infrared spectrum of a weakly bound complex of the $\mathrm{CN}$ radical is observed for the first time, specifically for the $\mathrm{CN}-\mathrm{Ne}$ complex. Infrared spectra of jet-cooled $\mathrm{CN}-\mathrm{Ne}$ are obtained principally in the $\mathrm{CN}$ overtone region at $\sim 2.5 \mu \mathrm{m}$ via an IR-UV double resonance scheme; analogous spectra are recorded in the fundamental $\mathrm{CN}$ stretch and second overtone regions. The IR spectra access various hindered internal rotor states of the complex with $n^{K}=0^{0}, 1^{1}$, and $1^{0}$. UV excitation in the $B^{2} \Sigma^{+}(\mathrm{v}=0)-X^{2} \Sigma^{+}(\mathrm{v}=2)$ spectral region promotes $\mathrm{CN}-\mathrm{Ne}$ to the excited $B\left(v_{\mathrm{CN}}=0\right)$ state. The energy difference between analogous transitions observed in the $B(\mathrm{v}=0)-X(\mathrm{v}$ $=2)$ and $B(\mathrm{v}=0)-X(\mathrm{v}=0)$ regions yields the pure $\mathrm{CN}$ overtone stretch of $\mathrm{CN}-\mathrm{Ne}\left(n^{K}=0^{0}\right)$ at $4058.6(1) \mathrm{cm}^{-1}$, which is unchanged from that of free $\mathrm{CN}$.

Rotationally structured bands of the $\mathrm{CN}-\mathrm{Ne}$ complex are identified at 4055.55(1), 4061.74(1), and 4064.32(1) $\mathrm{cm}^{-1}$ upon $\mathrm{CN}$ overtone excitation and are assigned as follows: $\left(v_{\mathrm{CN}}, n^{K}\right)=\left(2,0^{0}\right)-\left(0,1^{1}\right)$ (hot band), $\left(2,1^{1}\right)-\left(0,0^{0}\right)$, and $\left(2,1^{0}\right)-$ $\left(0,0^{0}\right)$. Rotational levels of the closely spaced $\left(2,1^{1}\right)$ and $\left(2,1^{0}\right)$ states of $\mathrm{CN}-\mathrm{Ne} X\left(v_{\mathrm{CN}}=2\right)$ accessed through $P$ - and $R$-branch lines in the IR spectra exhibit noticeable perturbations due to Coriolis coupling. A deperturbation analysis is carried out to extract accurate spectroscopic constants for the hindered internal rotor state and the magnitude of the $N$ dependent coupling.

In addition, the energetic ordering of the $n^{K}=0^{0}, 1^{1}$, and $1^{0}$ states and the spacings between them, specifically $\Delta \mathrm{E}$ $=3.1(1)$ and 2.6(1) $\mathrm{cm}^{-1}$ for $v_{\mathrm{CN}}=2$ and more limited data for other $v_{\mathrm{CN}}$, provide detailed information on the angular anisotropy of the $\mathrm{CN} X^{2} \Sigma^{+}+\mathrm{Ne}$ potential. The theoretical framework of Dubernet et al. ${ }^{18}$ for open-shell diatom-rare gas complexes is utilized to construct a bend- ing Hamiltonian and solve for its eigenvalues, which are compared with experimental spacings. The procedure is iterated to deduce radially averaged anisotropy parameters, $V(\theta)=V_{00}+V_{10} \cos \theta+V_{20}\left(3 \cos ^{2} \theta-1\right) / 2, \quad$ characterizing the anisotropic portion of the intermolecular potential. The weakly anisotropic potential favors a bent $\mathrm{CN}-\mathrm{Ne}$ configuration on average with only an $8 \mathrm{~cm}^{-1}$ barrier to internal rotation of $\mathrm{CN}$, giving rise to wide amplitude motion. High-level ab initio calculations of the $\mathrm{CN} X^{2} \Sigma^{+}+\mathrm{Ne}$ potential were carried out for comparison with the experimentally derived potential. The angular ab initio potential for $\mathrm{CN}-\mathrm{Ne}$, obtained by optimizing the radial potential at each angle, is in remarkably good agreement with experiment.

\section{ACKNOWLEDGMENTS}

The research at the University of Pennsylvania was supported by the Chemistry Division of the National Science Foundation. Acknowledgement is made to the Donors of the American Chemical Society Petroleum Research Fund for partial support of this research (T.A.S). T.A.S. also thanks Nadine Halberstadt for the computer code used to calculate probability amplitudes.

${ }^{1}$ R. J. Balla, K. H. Casleton, J. S. Adams, and L. Pasternack, J. Phys. Chem. 95, 8694 (1991).

${ }^{2}$ L. R. Copeland, F. Mohammad, M. Zahedi, D. H. Volman, and W. M. Jackson, J. Chem. Phys. 96, 5817 (1992).

${ }^{3}$ I. R. Sims, J. L. Queffelec, D. Travers, B. R. Rowe, L. B. Herbert, J. Karthauser, and I. W. M. Smith, Chem. Phys. Lett. 211, 461 (1993)

${ }^{4}$ D. L. Yang, T. Yu, N. S. Wang, and M. C. Lin, Chem. Phys. 160, 307 (1992).

${ }^{5}$ B. Atakan and J. Wolfrum, Chem. Phys. Lett. 186, 547 (1991).

${ }^{6}$ N. Balucani, O. Asvany, A. H. H. Chang, S. H. Lin, Y. T. Lee, R. I. Kaiser, and Y. Osamura, J. Chem. Phys. 113, 8643 (2000).

${ }^{7}$ Y. Georgievskii and S. J. Klippenstein, J. Phys. Chem. A 111, 3802 (2007).

${ }^{8}$ Y. Chen and M. C. Heaven, J. Chem. Phys. 109, 5171 (1998).

${ }^{9}$ S. Fei and M. C. Heaven, Proc. SPIE-Int. Soc. Opt. Eng. 1858, 286 (1993).

${ }^{10}$ J. Han, M. C. Heaven, U. Schnupf, and M. H. Alexander, J. Chem. Phys. 128, 104308 (2008).

${ }^{11}$ Y. Lin and M. C. Heaven, J. Chem. Phys. 94, 5765 (1991).

${ }^{12}$ J. Han, M. C. Heaven, and U. Schnupf, J. Chem. Phys. 128, 224309 (2008).

${ }^{13}$ A. L. Kaledin and M. C. Heaven, Chem. Phys. Lett. 347, 199 (2001).

${ }^{14}$ A. L. Kaledin, M. C. Heaven, and J. M. Bowman, J. Chem. Phys. 110, 10380 (1999).

${ }^{15}$ M. Yang and M. H. Alexander, J. Chem. Phys. 107, 7148 (1997).

${ }^{16}$ I. Vrabel, V. Lukes, V. Laurinc, and S. Biskupic, J. Phys. Chem. A 104, 96 (2000)

${ }^{17}$ R. Thomson and F. W. Dalby, Can. J. Phys. 46, 2815 (1968).

${ }^{18}$ M.-L. Dubernet, D. Flower, and J. M. Hutson, J. Chem. Phys. 94, 7602 (1991).

${ }^{19}$ M.-L. Dubernet and J. M. Hutson, J. Chem. Phys. 101, 1939 (1994).

${ }^{20} \mathrm{~J}$. M. Hutson, in Advances in Molecular Vibrations and Collision Dynamics, edited by J. M. Bowman and M. A. Ratner (JAI Press, Greenwich, CT, 1991) Vol. 1A, pp. 1

${ }^{21}$ J. Larsen, D. Madsen, J.-A. Poulsen, T. D. Poulsen, S. R. Keiding, and J. Thogersen, J. Chem. Phys. 116, 7997 (2002).

${ }^{22}$ A. C. Moskun and S. E. Bradforth, J. Chem. Phys. 119, 4500 (2003).

${ }^{23}$ R. J. Lewis, Sr., Sax's Dangerous Properties of Industrial Materials, 11th ed. (Wiley, New York, 2004) Vols. 1-3.

${ }^{24}$ M. Lambert, B. Callen, H. Dugan, S. V. Filseth, F. J. Morgan, and C. M. Sadowski, Chem. Phys. Lett. 139, 45 (1987).

${ }^{25}$ M. A. O'Halloran, H. Joswig, and R. N. Zare, J. Chem. Phys. 87, 303 (1987) 
${ }^{26}$ G. Herzberg, Molecular Spectra and Molecular Structure, 2nd ed. (Van Nostrand Reinhold, New York, 1950).

${ }^{27}$ F. A. Jenkins, Phys. Rev. 31, 539 (1928).

${ }^{28}$ See supplementary material at http://dx.doi.org/10.1063/1.3586810 for details of the theoretical methods and hindered rotor analysis including the correlation diagram.

${ }^{29}$ H.-J. Werner, P. J. Knowles, F. R. Manby, M. Schütz, et al., Molpro, version 2010.1, a package of ab initio programs, 2010, see http://www.molpro.net.

${ }^{30}$ D. Cerny, R. Bacis, G. Guelachvili, and F. Roux, J. Mol. Spectrosc. 73, 154 (1978).

${ }^{31}$ R. Fei, D. E. Adelman, T. Carrington, C. H. Dugan, and S. V. Filseth, Chem. Phys. Lett. 232, 547 (1995).
${ }^{32}$ K. M. Hickson, C. M. Sadowski, and I. W. M. Smith, Chem. Phys. Lett. 372, 443 (2003).

${ }^{33}$ PGopher, a program for simulating rotational structure, C. M. Western, University of Bristol, available at http://pgopher.chm.bris.ac.uk.

${ }^{34}$ C. M. Lovejoy, J. M. Hutson, and D. J. Nesbitt, J. Chem. Phys. 97, 8009 (1992).

${ }^{35}$ C. M. Lovejoy and D. J. Nesbitt, J. Chem. Phys. 91, 2790 (1989).

${ }^{36}$ M. D. Schuder, D. D. Nelson, and D. J. Nesbitt, J. Chem. Phys. 94, 5796 (1991).

${ }^{37}$ D. J. Nesbitt, C. M. Lovejoy, T. G. Lindeman, S. V. Oneil, and D. C. Clary, J. Chem. Phys. 91, 722 (1989). 